Article

\title{
On Squeezed Flow of Jeffrey Nanofluid between Two Parallel Disks
}

\author{
Tasawar Hayat ${ }^{1,2}$, Tehseen Abbas ${ }^{1, *}$, Muhammad Ayub ${ }^{1}$, Taseer Muhammad ${ }^{1}$ \\ and Ahmed Alsaedi ${ }^{2}$ \\ 1 Department of Mathematics, Quaid-I-Azam University, Islamabad 44000, Pakistan; \\ fmgpak@gmail.com (T.H.); mayub@qau.edu.pk (M.A.); taseer@math.qau.edu.pk (T.M.) \\ 2 Nonlinear Analysis and Applied Mathematics (NAAM) Research Group, Department of Mathematics, \\ Faculty of Science, King Abdulaziz University, Jeddah 21589, Saudi Arabia; aalsaedi@hotmail.com \\ * Correspondence: tehseen@math.qau.edu.pk; Tel.: +92-51-9064-2172
}

Academic Editor: Rahmat Ellahi

Received: 7 September 2016 ; Accepted: 31 October 2016 ; Published: 11 November 2016

\begin{abstract}
The present communication examines the magnetohydrodynamic (MHD) squeezing flow of Jeffrey nanofluid between two parallel disks. Constitutive relations of Jeffrey fluid are employed in the problem development. Heat and mass transfer aspects are examined in the presence of thermophoresis and Brownian motion. Jeffrey fluid subject to time dependent applied magnetic field is conducted. Suitable variables lead to a strong nonlinear system. The resulting systems are computed via homotopic approach. The behaviors of several pertinent parameters are analyzed through graphs and numerical data. Skin friction coefficient and heat and mass transfer rates are numerically examined.
\end{abstract}

Keywords: squeezing flow; Jeffrey fluid; nanoparticles; magnetic field

\section{Introduction}

The homogenous mixture of ultrafine nanometer-sized particles and convectional heat transfer base liquids is termed as nanofluid. Nanomaterials have a key role in the industrial and engineering processes like processing of coolants for the nuclear reactors, transformer coolant and radiation therapy in cancer treatment etc. Furthermore, the magneto-nanofluid is very helpful in various sectors including sterilizing devices, oil recovery from the underground reservoirs, gastric medications, and tumor elimination with hyperthermia. The small sized nanoparticles (which are mostly metallic, nonmetallic, metal-oxides) are good thermal conductors. For this reason, the nanofluid in comparison to the base fluid has greater thermal efficiency. Choi [1] proposed the idea of nanofluid. He argued that the addition of nanoparticles into the base fluid enhances the thermal performance of base fluid. Buongiorno [2] provided expressions including thermophoresis and Brownian motion. Later on, numerous researchers discussed the flows of nanofluid under different geometries. The relevant literature can be seen through the investigations [3-20] and several studies therein.

Squeezing flow between the parallel disks has received the attention of recent researchers due to widespread applications of such flows in various mechanical engineering disciplines. The flow is generated because of two parallel approaching surfaces in relative motion. The parallel approaching surfaces phenomena along with the relative motion is mostly used by the engineers in the modeling of flow of oil in bearings, determination of capacity of load-bearings, compression and injection modeling, etc. (see [21,22]). Stefan [23] reported the squeezing flow for lubrication approximation. Domairry and Aziz [24] studied magnetohydrodynamic squeezing flow of viscous liquid bounded by parallel disks. Siddiqui et al. [25] examined squeezing flow subject to an applied magnetic field. Rashidi et al. [26] performed an analysis of hydrodynamic squeezing flow by developing series solutions. Some other investigations on squeezing flow can be seen in the studies [27-30]. 
The prime interest in the present communication is to venture further into the regime of the squeezing flow of non-Newtonian nanofluid. Therefore, the explicit contribution here is as follows: firstly, to formulate the relevant problem for constitutive relations of the Jeffrey fluid model; secondly, to analyze Brownian motion and thermophoresis; thirdly, to consider magnetohydrodynamics of nanofluid; and fourthly, to entertain the idea of permeable characteristics of lower disks. The upper impermeable disk moves towards the lower disk with time-dependent velocity. Problem formulation is made through small magnetic Reynolds number approximation. The homotopy analysis technique (HAM) [31-40] is applied to obtain the convergent solutions of the governing equations. The present study has been arranged as follows. The next section presents problem development. Section 3 depicts the development convergent series solutions. Analysis for convergence and discussion have been examined in Sections 4 and 5, respectively. Section 6 gives the main outcomes of the present study. Note that the considered Jeffrey fluid, although capturing the salient features of relaxation and retardation time, is not able to predict the shear thinning/shear thickening and normal stress effects.

\section{Formulation}

Consider magnetohydrodynamic squeezing flow of a Jeffrey nanofluid between the two parallel disks. The distance between the parallel disks is $h(t)=H(1-\alpha t)^{1 / 2}$. The upper disk is at $z=h(t)$, whereas the lower permeable disk is at $z=0$. A magnetic field $B(t)=B_{0}(1-\alpha t)^{-1 / 2}$ is taken transverse to the flow. Here, the induced magnetic field is neglected for a small magnetic Reynolds number [41-43]. Brownian motion and thermophoresis phenomena are accounted. The governing equations for Jeffrey nanofluid are differences in traffic flow

$$
\begin{aligned}
& \frac{\partial u}{\partial r}+\frac{u}{r}+\frac{\partial w}{\partial z}=0 \\
& \left(\frac{\partial u}{\partial t}+u \frac{\partial u}{\partial r}+w \frac{\partial u}{\partial z}\right)=-\frac{1}{\rho} \frac{\partial p}{\partial r}+\frac{v}{1+\lambda_{1}}\left(\frac{\partial^{2} u}{\partial r^{2}}+\frac{\partial^{2} u}{\partial z^{2}}+\frac{1}{r} \frac{\partial u}{\partial r}-\frac{u}{r^{2}}\right) \\
& +\frac{v \lambda_{2}}{1+\lambda_{1}}\left(\begin{array}{c}
\frac{\partial^{3} u}{\partial t \partial z^{2}}+2 \frac{\partial^{3} u}{\partial t \partial r^{2}}+\frac{2}{r} \frac{\partial^{2} u}{\partial t \partial z}+\frac{\partial^{3} w}{\partial t \partial r \partial z} \\
-\frac{2}{r^{2}} \frac{\partial u}{\partial t}+\frac{\partial u}{\partial r}\left(\frac{\partial^{2} u}{\partial r^{2}}-2 \frac{u}{r^{2}}\right)+\frac{\partial w}{\partial r} \frac{\partial^{2} u}{\partial z \partial r} \\
+u\left(\frac{\partial^{3} u}{\partial r^{3}}+\frac{\partial^{3} w}{\partial r^{2} \partial z}+\frac{\partial^{3} u}{\partial r \partial z^{2}}+2 \frac{u}{r^{3}}\right) \\
+w\left(\frac{\partial^{3} u}{\partial z \partial r^{2}}+\frac{2}{r} \frac{\partial^{2} u}{\partial z \partial r}+\frac{\partial^{3} w}{\partial z^{3}}+\frac{\partial^{2} w}{\partial r \partial z}\right) \\
+\frac{\partial u}{\partial z}\left(\frac{\partial^{2} w}{\partial r^{2}}-2 \frac{w}{r^{2}}+\frac{\partial^{2} u}{\partial z \partial r}\right) \\
+\frac{\partial w}{\partial z}\left(\frac{\partial^{2} u}{\partial z^{2}}+\frac{\partial^{2} w}{\partial r \partial z}\right)+\frac{2}{r} \frac{\partial^{2} u}{\partial r^{2}}
\end{array}\right)-\frac{\sigma B^{2}}{\rho} u, \\
& \left(\frac{\partial w}{\partial t}+u \frac{\partial w}{\partial r}+w \frac{\partial w}{\partial z}\right)=-\frac{1}{\rho} \frac{\partial p}{\partial z}+\frac{v}{1+\lambda_{1}}\left(\frac{\partial^{2} w}{\partial r^{2}}+\frac{\partial^{2} w}{\partial z^{2}}+\frac{1}{r} \frac{\partial w}{\partial r}\right) \\
& +\frac{v \lambda_{2}}{1+\lambda_{1}}\left(\begin{array}{c}
\frac{\partial^{3} w}{\partial r \partial t \partial z}+\frac{\partial^{3} w}{\partial t \partial r^{2}}+2 \frac{\partial^{3} u}{\partial r \partial z^{2}} \\
+2 \frac{\partial u}{\partial z} \frac{\partial^{2} w}{r \partial z}+\frac{\partial u}{\partial r}\left(\frac{\partial^{2} u}{\partial r \partial z}+\frac{\partial^{2} w}{\partial r^{2}}\right) \\
+u\left(\frac{\partial^{3} w}{\partial r^{2} \partial z}+\frac{\partial^{3} u}{\partial r^{3}}\right)+\frac{\partial w}{\partial r}\left(\frac{\partial^{2} u}{\partial z^{2}}+\frac{\partial^{3} u}{\partial t \partial z^{2}}\right) \\
+w\left(\frac{\partial^{3} u}{\partial r \partial z^{2}}+\frac{\partial^{3} w}{\partial r^{2} \partial z}+\frac{\partial^{3} w}{\partial z^{3}}+\frac{1}{r} \frac{\partial^{2} u}{\partial z^{2}}+\frac{1}{r} \frac{\partial^{2} w}{\partial z \partial r}\right) \\
+\frac{u}{r}\left(\frac{\partial^{2} u}{\partial r \partial z}+\frac{\partial^{2} w}{\partial r^{2}}\right)+\frac{1}{r}\left(\frac{\partial^{2} u}{\partial t \partial z}+\frac{\partial^{2} w}{\partial t \partial r}\right)
\end{array}\right), \\
& \frac{\partial T}{\partial t}+u \frac{\partial T}{\partial r}+w \frac{\partial T}{\partial z}=\alpha\left(\frac{\partial^{2} T}{\partial r^{2}}+\frac{1}{r} \frac{\partial T}{\partial r}+\frac{\partial^{2} T}{\partial z^{2}}\right)+\tau\left(\begin{array}{c}
D_{B}\left(\frac{\partial C}{\partial r} \frac{\partial T}{\partial r}+\frac{\partial C}{\partial z} \frac{\partial T}{\partial z}\right) \\
+\frac{D_{T}}{T_{m}}\left(\left(\frac{\partial T}{\partial r}\right)^{2}+\left(\frac{\partial T}{\partial z}\right)^{2}\right)
\end{array}\right), \\
& \frac{\partial C}{\partial t}+u \frac{\partial C}{\partial r}+w \frac{\partial C}{\partial z}=D_{B}\left(\frac{\partial^{2} C}{\partial r^{2}}+\frac{1}{r} \frac{\partial C}{\partial r}+\frac{\partial^{2} C}{\partial z^{2}}\right)+\frac{D_{T}}{T_{m}}\left(\frac{\partial^{2} T}{\partial r^{2}}+\frac{1}{r} \frac{\partial T}{\partial r}+\frac{\partial^{2} T}{\partial z^{2}}\right),
\end{aligned}
$$


with the associated boundary conditions

$$
\left.\begin{array}{l}
u=0, w=-w_{0}, T=T_{w}, C=C_{w} \text { at } z=0 \\
u=0, w=\frac{\partial h}{\partial t}, T=T_{h}, C=C_{h} \text { at } z=h(t)
\end{array}\right\}
$$

Here, $u$ and $w$ denote the velocity components along the $r$ - and $z$-directions, respectively, $p$ the pressure, $v=\mu / \rho$ the kinematic viscosity, $\mu$ the dynamic viscosity, $\rho$ the density of base fluid, $\sigma$ the electrical conductivity, $\lambda_{1}$ the ratio of relaxation and retardation times, $\lambda_{2}$ the retardation time, respectively, $T$ the temperature, $\tau=(\rho c)_{p} /(\rho c)_{f}$ the ratio of effective heat capacity of nanoparticles and heat capacity of fluid, $(\rho c)_{p}$ the effective heat capacity of nanoparticles, $(\rho c)_{f}$ the heat capacity of fluid, $C$ the concentration, $T_{m}$ the mean fluid temperature, $\alpha=k /(\rho c)_{f}$ the thermal diffusivity, $k$ the thermal conductivity, $D_{B}$ the Brownian diffusion coefficient and $D_{T}$ the thermophoresis diffusion coefficient. Consider

$$
\begin{gathered}
u=\frac{\alpha r}{2(1-\alpha t)} f^{\prime}(\eta), w=-\frac{\alpha H}{\sqrt{1-\alpha t}} f(\eta), \eta=\frac{z}{H \sqrt{1-\alpha t}}, \\
\theta(\eta)=\frac{T-T_{h}}{T_{w}-T_{h}}, \phi(\eta)=\frac{C-C_{h}}{C_{w}-C_{h}} .
\end{gathered}
$$

Equations (2)-(6) after elimination of pressure gradient yield

$$
\begin{gathered}
f^{i v}-S q\left(1+\lambda_{1}\right)\left(\eta f^{\prime \prime \prime}+3 f^{\prime \prime}-2 f f^{\prime \prime \prime}\right)+\frac{\beta}{2}\left(\eta f^{v}+5 f^{i v}+f^{\prime \prime} f^{\prime \prime \prime}-3 f^{\prime} f^{i v}\right)-M^{2}\left(1+\lambda_{1}\right) f^{\prime \prime}=0, \\
\theta^{\prime \prime}+\operatorname{Pr} S q\left(f \theta^{\prime}-\eta \theta^{\prime}\right)+\operatorname{Pr} N b \theta^{\prime} \phi^{\prime}+\operatorname{Pr} N t \theta^{\prime 2}=0, \\
\phi^{\prime \prime}+\operatorname{Pr} L e S q\left(f \phi^{\prime}-\eta \phi^{\prime}\right)+\frac{N t}{N b} \theta^{\prime \prime}=0, \\
f(0)=S, f^{\prime}(0)=0, \theta(0)=1, \phi(0)=1, \\
f(1)=\frac{1}{2}, f^{\prime}(1)=0, \quad \theta(1)=0, \quad \phi(1)=0 .
\end{gathered}
$$

Here, $\mathrm{Pr}$ denotes the Prandtl number, Le the Lewis number, $\mathrm{Nb}$ Brownian motion parameter, $S$ the suction/blowing parameter, $N t$ the thermophoresis parameter, $\beta$ the Deborah number, $M$ the Hartman number and $S q$ the squeezing parameter. These quantities are expressed as follows:

$$
\begin{aligned}
& P r=\frac{v}{\alpha}, L e=\frac{\alpha}{D_{B}}, N b=\frac{\tau D_{B}}{v}\left(C_{w}-C_{h}\right), S=\frac{w_{0}}{\alpha H}, \\
& S q=\frac{\alpha H^{2}}{2 v}, N t=\frac{\tau D_{T}}{v T_{m}}\left(T_{w}-T_{h}\right), M=H B_{0} \sqrt{\frac{\sigma}{\mu}}, \beta=\frac{\lambda_{2} \alpha}{1-\alpha t} .
\end{aligned}
$$

Expressions of skin frictions corresponding to lower and upper disks are

$$
C_{f 1}=\frac{\left.\tau_{r z}\right|_{z=0}}{\rho\left(\frac{\alpha H}{2(1-\alpha t)^{1 / 2}}\right)^{2}},
$$

and

$$
C_{f 2}=\frac{\left.\tau_{r z}\right|_{z=h(t)}}{\rho\left(\frac{\alpha H}{2(1-\alpha t)^{1 / 2}}\right)^{2}}
$$

with

$$
\begin{aligned}
\tau_{r z}= & \frac{\mu}{1+\lambda_{1}}\left(\frac{\partial u}{\partial z}+\frac{\partial w}{\partial r}\right) \\
& +\frac{\lambda_{2}}{1+\lambda_{1}}\left(\frac{\partial^{2} u}{\partial t \partial z}+\frac{\partial^{2} w}{\partial t \partial r}+u\left(\frac{\partial^{2} u}{\partial r \partial z}+\frac{\partial^{2} w}{\partial r^{2}}\right)+w\left(\frac{\partial^{2} u}{\partial z^{2}}+\frac{\partial^{2} w}{\partial z \partial r}\right)\right) .
\end{aligned}
$$


The dimensionless forms of skin friction coefficients are

$$
\frac{H^{2}}{r^{2}} \operatorname{Re}_{r} C_{f 1}=\left(1+\frac{3}{2} \beta\right) f^{\prime \prime}(0),
$$

and

$$
\frac{H^{2}}{r^{2}} \operatorname{Re}_{r} C_{f 2}=\left(1+\frac{3}{2} \beta\right) f^{\prime \prime}(1),
$$

where

$$
R e_{r}^{-1}=\frac{2 v}{r \alpha H\left(1+\lambda_{1}\right)(1-\alpha t)^{1 / 2}} .
$$

Local Nusselt numbers at lower and upper disks are given by

$$
N u_{r 1}=-\left.\frac{H}{\left(T_{w}-T_{h}\right)} \frac{\partial T}{\partial z}\right|_{z=0}=-\frac{1}{\sqrt{1-\alpha t}} \theta^{\prime}(0),
$$

and

$$
N u_{r 2}=-\left.\frac{H}{\left(T_{w}-T_{h}\right)} \frac{\partial T}{\partial z}\right|_{z=h(t)}=-\frac{1}{\sqrt{1-\alpha t}} \theta^{\prime}(1) .
$$

Local Sherwood numbers at lower and upper disks can be expressed as follows:

$$
S h_{r_{1}}=-\left.\frac{H}{\left(C_{w}-C_{h}\right)} \frac{\partial C}{\partial z}\right|_{z=0}=-\frac{1}{\sqrt{1-\alpha t}} \phi^{\prime}(0),
$$

and

$$
S h_{r_{2}}=-\left.\frac{H}{\left(C_{w}-C_{h}\right)} \frac{\partial C}{\partial z}\right|_{z=h(t)}=-\frac{1}{\sqrt{1-\alpha t}} \phi^{\prime}(1) .
$$

\section{Homotopic Solutions}

\subsection{Zeroth-Order Deformation}

Here, we construct the convergent series solutions of the incoming nonlinear systems. For these, the initial approximation and auxiliary linear operators are taken in the form

$$
\begin{gathered}
f_{0}(\eta)=(-1+2 S) \eta^{3}-\frac{1}{2}(-3+6 S) \eta^{2}+S, \theta_{0}(\eta)=1-\eta, \phi_{0}(\eta)=1-\eta, \\
\mathcal{L}_{f}(f)=\frac{d^{4} f}{d \eta^{4}}, \mathcal{L}_{\theta}(\theta)=\frac{d^{2} \theta}{d \eta^{2}}, \mathcal{L}_{\phi}(\phi)=\frac{d^{2} \phi}{d \eta^{2}}
\end{gathered}
$$

with the properties

$$
\begin{gathered}
\mathcal{L}_{f}\left[B_{1}^{*}+B_{2}^{*} \eta+B_{3}^{*} \eta^{2}+B_{4}^{*} \eta^{3}\right]=0, \\
\mathcal{L}_{\theta}\left[B_{5}^{*}+B_{6}^{*} \eta\right]=0, \\
\mathcal{L}_{\phi}\left[B_{7}^{*}+B_{8}^{*} \eta\right]=0 .
\end{gathered}
$$

Here, $B_{i}^{*}(i=1-8)$ are the arbitrary constants. The zeroth-order deformation statements are

$$
\begin{gathered}
(1-\mathrm{P}) \mathcal{L}_{f}\left[\widehat{f}(\eta ; \mathrm{P})-f_{0}(\eta)\right]=\mathrm{P} \hbar_{f} \mathcal{N}_{f}[\widehat{f}(\eta ; \mathrm{P})], \\
(1-\mathrm{P}) \mathcal{L}_{\theta}\left[\widehat{\theta}(\eta ; \mathrm{P})-\theta_{0}(\eta)\right]=\mathrm{P} \hbar_{\theta} \mathcal{N}_{\theta}[\widehat{f}(\eta ; \mathrm{P}), \widehat{\theta}(\eta ; \mathrm{P}), \widehat{\phi}(\eta ; \mathrm{P})], \\
(1-\mathrm{P}) \mathcal{L}_{\phi}\left[\widehat{\phi}(\eta ; \mathrm{P})-\phi_{0}(\eta)\right]=\mathrm{P} \hbar_{\phi} \mathcal{N}_{\phi}[\widehat{f}(\eta ; \mathrm{P}), \widehat{\theta}(\eta ; \mathrm{P}), \widehat{\phi}(\eta ; \mathrm{P})],
\end{gathered}
$$




$$
\begin{aligned}
& \widehat{f}(0 ; \mathrm{P})=S, \widehat{f}^{\prime}(0 ; \mathrm{P})=0, \widehat{\theta}(0 ; \mathrm{P})=1, \widehat{\phi}(0 ; \mathrm{P})=1, \\
& \widehat{f}(1 ; \mathrm{P})=\frac{1}{2}, \widehat{f}^{\prime}(1 ; \mathrm{P})=0, \widehat{\theta}(1 ; \mathrm{P})=0, \widehat{\phi}(1 ; \mathrm{P})=0, \\
& \mathcal{N}_{f}[\widehat{f}(\eta, \mathrm{P})]=\frac{\partial^{4} \widehat{f}(\eta ; \mathrm{P})}{\partial \eta^{4}}-S q\left(1+\lambda_{1}\right)\left(\begin{array}{c}
\eta \frac{\partial^{3} \widehat{f}(\eta ; \mathrm{F})}{\partial \eta^{3}}+3 \frac{\partial^{2} \widehat{f}(\eta ; \mathrm{P})}{\partial \eta^{2}} \\
-2 \frac{\partial \hat{f}(\eta ; \mathrm{P})}{\partial \eta} \frac{\partial^{3} \widehat{f}(\eta ; \mathrm{P})}{\partial \eta^{3}}
\end{array}\right) \\
& +\frac{\beta}{2}\left(\begin{array}{c}
\eta \frac{\partial^{5} \hat{f}(\eta ; \mathrm{P})}{\partial \eta^{5}}+5 \frac{\partial^{4} \hat{f}(\eta ; \mathrm{F})}{\partial \eta^{4}} \\
+\frac{\partial^{2} \widehat{f}(\eta ; \mathrm{P})}{\partial \eta^{2}} \frac{\partial^{3} \hat{f}(\eta ; \mathrm{P})}{\partial \eta^{3}}-3 \frac{\partial \hat{f}(\eta ; \mathrm{P})}{\partial \eta} \frac{\partial^{4} \widehat{f}(\eta ; \mathrm{P})}{\partial \eta^{4}}
\end{array}\right)-M^{2}\left(1+\lambda_{1}\right) \frac{\partial^{2} \widehat{f}(\eta ; \mathrm{P})}{\partial \eta^{2}}, \\
& \mathcal{N}_{\theta}[\widehat{f}(\eta, \mathrm{P}), \widehat{\theta}(\eta ; \mathrm{P}), \widehat{\phi}(\eta ; \mathrm{P})]=\frac{1}{P r} \frac{\partial^{2} \widehat{\theta}(\eta ; \mathrm{P})}{\partial \eta^{2}}+S q\left(\widehat{f}(\eta ; \mathrm{P}) \frac{\partial \widehat{\theta}(\eta ; \mathrm{P})}{\partial \eta}-\eta \frac{\partial \widehat{\theta}(\eta ; \mathrm{P})}{\partial \eta}\right) \\
& +N b \frac{\partial \widehat{\theta}(\eta ; \mathrm{P})}{\partial \eta} \frac{\partial \widehat{\phi}(\eta ; \mathrm{P})}{\partial \eta}+N t\left(\frac{\partial \widehat{\theta}(\eta ; \mathrm{P})}{\partial \eta}\right)^{2}, \\
& \mathcal{N}_{\phi}[\widehat{f}(\eta, \mathrm{P}), \widehat{\theta}(\eta ; \mathrm{P}), \widehat{\phi}(\eta ; \mathrm{P})]=\frac{\partial^{2} \widehat{\phi}(\eta ; \mathrm{P})}{\partial \eta^{2}}+\operatorname{PrLeSq} \widehat{f}(\eta ; \mathrm{P}) \frac{\partial \widehat{\phi}(\eta ; \mathrm{P})}{\partial \eta} \\
& -\operatorname{PrLeSq\eta } \frac{\partial \widehat{\phi}(\eta ; \mathrm{P})}{\partial \eta}+\frac{N t}{N b} \frac{\partial^{2} \widehat{\theta}(\eta ; \mathrm{P})}{\partial \eta^{2}} \text {, }
\end{aligned}
$$

Here, $\mathbf{P} \in[0,1]$ indicates the embedding parameter and $\hbar_{f}, \hbar_{\theta}$ and $\hbar_{\phi}$ the non-zero auxiliary parameters.

3.2. $m^{\text {th }}$-Order Deformation Equations

$$
\begin{gathered}
\mathcal{L}_{f}\left[f_{m}(\eta)-\chi_{m} f_{m-1}(\eta)\right]=\hbar_{f} \mathcal{R}_{m}^{f}(\eta), \\
\mathcal{L}_{\theta}\left[\theta_{m}(\eta)-\chi_{m} \theta_{m-1}(\eta)\right]=\hbar_{\theta} \mathcal{R}_{m}^{\theta}(\eta), \\
\mathcal{L}_{\phi}\left[\phi_{m}(\eta)-\chi_{m} \phi_{m-1}(\eta)\right]=\hbar_{\phi} \mathcal{R}_{m}^{\phi}(\eta), \\
f_{m}(0)=f_{m}^{\prime}(0)=0, \theta_{m}(0)=\phi_{m}(0)=0, \\
f_{m}(1)=f_{m}^{\prime}(1)=0, \phi_{m}(1)=\theta_{m}(1)=0, \\
\mathcal{R}_{m}^{f}(\eta)=f_{m-1}^{\prime v}-S q\left(1+\lambda_{1}\right)\left(\eta f_{m-1}^{\prime \prime \prime}+3 f_{m-1}^{\prime \prime}-2 \sum_{k=0}^{m-1} f_{m-1-k} f_{k}^{\prime \prime \prime}\right) \\
+\frac{\beta}{2}\left(\eta f_{m-1}^{v}+5 f_{m-1}^{i v}+\sum_{k=0}^{m-1} f_{m-1-k}^{\prime \prime} f_{k}^{\prime \prime \prime}-3 \sum_{k=0}^{m-1} f_{m-1-k}^{\prime} f_{k}^{i v}\right)-M^{2}\left(1+\lambda_{1}\right) f_{m-1}^{\prime \prime}, \\
\mathcal{R}_{m}^{\theta}(\eta)=\frac{1}{P r} \theta_{m-1}^{\prime \prime}+S q\left(\sum_{k=0}^{m-1} f_{m-1-k} \theta_{k}^{\prime}-\eta \theta_{m-1}^{\prime}\right)+N b \sum_{k=0}^{m-1} \theta_{m-1-k}^{\prime} \phi_{k}^{\prime}+N t \sum_{k=0}^{m-1} \theta_{m-1-k}^{\prime} \theta_{k}^{\prime}, \\
\mathcal{R}_{m}^{\phi}(\eta)=\phi_{m-1}^{\prime \prime}+\frac{N t}{N b} \theta_{m-1}^{\prime \prime}+\operatorname{PrLeSq}\left(\sum_{k=0}^{m-1} f_{m-1-k} \phi_{k}^{\prime}-\eta \phi_{m-1}^{\prime}\right), \\
\chi_{m}=\left\{\begin{array}{l}
m \leq 1, \\
1, m>1 .
\end{array}\right.
\end{gathered}
$$

The general solutions $\left(f_{m}, \theta_{m}, \phi_{m}\right)$ consisting of the special solutions $\left(f_{m}^{*}, \theta_{m}^{*}, \phi_{m}^{*}\right)$ are

$$
\begin{gathered}
f_{m}(\eta)=f_{m}^{*}(\eta)+B_{1}^{*}+B_{2}^{*} \eta+B_{3}^{*} \eta^{2}+B_{4} \eta^{3}, \\
\theta_{m}(\eta)=\theta_{m}^{*}(\eta)+B_{5}^{*}+B_{6}^{*} \eta, \\
\phi_{m}(\eta)=\phi_{m}^{*}(\eta)+B_{7}^{*}+B_{8}^{*} \eta,
\end{gathered}
$$


where the constants $B_{i}^{*}(i=1-8)$ are computed through the boundary conditions (41) and (42) with values

$$
\left.\begin{array}{c}
B_{1}^{*}=\left.f_{m}^{*}(\eta)\right|_{\eta=0}, B_{2}^{*}=\left.\frac{\partial f_{m}^{*}(\eta)}{\partial \eta}\right|_{\eta=0}, \\
B_{3}^{*}=-\left.3 f_{m}^{*}(\eta)\right|_{\eta=1}+\left.\frac{\partial f_{m}^{*}(\eta)}{\eta \eta}\right|_{\eta=1}-3 B_{1}^{*}-2 B_{2}^{*}, \\
B_{4}^{*}=\left.2 f_{m}^{*}(\eta)\right|_{\eta=1}-\left.\frac{\partial f_{m}^{*}(\eta)}{\partial \eta}\right|_{\eta=1}+2 B_{1}^{*}+B_{2}^{*}, \\
B_{5}^{*}=-\left.\theta_{m}^{*}(\eta)\right|_{\eta=0}, B_{6}^{*}=\left.\theta_{m}^{*}(\eta)\right|_{\eta=0}-\left.\theta_{m}^{*}(\eta)\right|_{\eta=1}, \\
B_{7}^{*}=-\left.\phi_{m}^{*}(\eta)\right|_{\eta=0}, B_{8}^{*}=\left.\phi_{m}^{*}(\eta)\right|_{\eta=0}-\left.\phi_{m}^{*}(\eta)\right|_{\eta=1}
\end{array}\right\}
$$

\section{Convergence Analysis}

Clearly, the approximate series solutions involve the nonzero auxiliary parameters $\hbar_{f}, \hbar_{\theta}$ and $\hbar_{\phi}$. To get the appropriate values of $\hbar_{f}, \hbar_{\theta}$ and $\hbar_{\phi}$, the $\hbar$-curves are plotted at 20th order of deformations. Figures 1 and 2 clearly show that the convergence zone exists inside the ranges $-1.30 \leq \hbar_{f} \leq-0.15$, $-1.45 \leq \hbar_{\theta} \leq-0.25$ and $-1.40 \leq \hbar_{\phi} \leq-0.20$ for lower disk case $(\eta=0)$ and $-1.10 \leq \hbar_{f} \leq-0.15$, $-1.35 \leq \hbar_{\theta} \leq-0.25$ and $-1.35 \leq \hbar_{\phi} \leq-0.10$ for upper disk case $(\eta=1)$. Table 1 depicts that 16 th order of deformations is sufficient for convergent homotopic solutions for lower disk, whereas the 18th order of deformations is necessary for convergent homotopic solutions regarding upper disks (see Table 2).

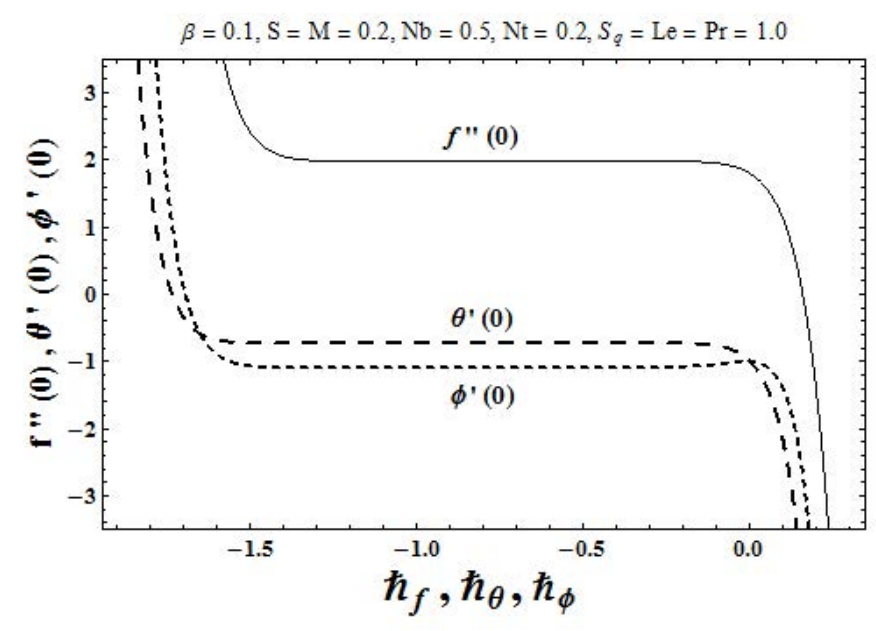

Figure 1. $\hbar$-Curves for $f, \theta$ and $\phi$ at the lower disk.

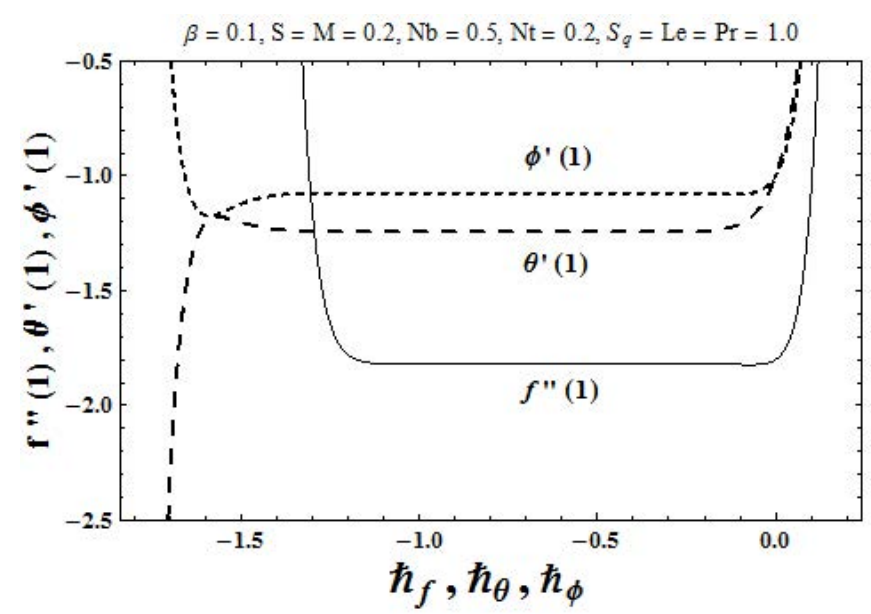

Figure 2. $\hbar$-Curves for $f, \theta$ and $\phi$ at the upper disk. 
Table 1. HAM solution convergence at the lower disk when $S=0.2, M=N t=0.2, N b=0.5$ and $S q=L e=\operatorname{Pr}=1.0$.

\begin{tabular}{cccc}
\hline Order of Deformations & $f^{\prime \prime}(\mathbf{0})$ & $-\boldsymbol{\theta}^{\prime}(\mathbf{0})$ & $-\boldsymbol{\phi}^{\prime}(\mathbf{0})$ \\
\hline 1 & 1.90121 & 0.08358 & 0.98916 \\
5 & 1.98026 & 0.72074 & 1.07442 \\
10 & 1.98313 & 0.71628 & 1.09068 \\
16 & 1.98318 & 0.71623 & 1.09150 \\
25 & 1.98318 & 0.71623 & 1.09150 \\
35 & 1.98318 & 0.71623 & 1.09150 \\
50 & 1.98318 & 0.71623 & 1.09150 \\
\hline
\end{tabular}

Table 2. HAM solution convergence at the upper disk when $S=0.2, M=N t=0.2, N b=0.5$ and $S q=L e=\operatorname{Pr}=1.0$.

\begin{tabular}{cccc}
\hline Order of Deformations & $-f^{\prime \prime}(\mathbf{1})$ & $-\theta^{\prime}(\mathbf{1})$ & $-\boldsymbol{\phi}^{\prime}(\mathbf{1})$ \\
\hline 1 & 1.82591 & 1.11083 & 1.06417 \\
5 & 1.82053 & 1.23444 & 1.08069 \\
10 & 1.81966 & 1.24300 & 1.07502 \\
18 & 1.81965 & 1.24304 & 1.07475 \\
25 & 1.81965 & 1.24304 & 1.07475 \\
35 & 1.81965 & 1.24304 & 1.07475 \\
50 & 1.81965 & 1.24304 & 1.07475 \\
\hline
\end{tabular}

\section{Discussion}

This portion explores the effects of various pertinent parameters including Deborah number $(\beta)$, Lewis number $(L e)$, Brownian motion parameter $(\mathrm{Nb})$, Prandtl number $(\mathrm{Pr})$, thermophoresis parameter $(N t)$ and squeezing parameter $(S q)$ on temperature $\theta(\eta)$ and concentration $\phi(\eta)$ profiles. Figure 3 shows the the impact of Deborah number $(\beta)$ on the temperature field $\theta(\eta)$. It is observed that the temperature field $\theta(\eta)$ decreases with the increase in the Deborah number $(\beta)$. Figure 4 illustrates the impact of Brownian motion parameter $(\mathrm{Nb})$ on temperature field $\theta(\eta)$. Here, temperature field $\theta(\eta)$ is increased by enhancing Brownian motion parameter. Variation of thermophoresis parameter $(N t)$ on temperature field $\theta(\eta)$ is sketched in Figure 5. Larger values of thermophoresis parameter $(N t)$ show higher temperature fields. Physically larger $(N t)$ causes an enhancement in temperature distribution. This is because of a stronger thermophoretic impact. Figure 6 shows temperature against $P r$. Lower temperature is noticed for larger $P r$. Figure 7 indicates that larger squeezing parameter $(S q)$ guarantees a decay in temperature $\theta(\eta)$. Figure 8 elucidates the impact of Deborah number $(\beta)$ on the concentration profile $\phi(\eta)$. The concentration field $\phi(\eta)$ is decreased by increasing the Deborah number $(\beta)$. Figure 9 shows the impact of Brownian motion parameter $(\mathrm{Nb})$ on concentration profile $\phi(\eta)$. Concentration profile is reduced for larger values of Brownian motion parameter $(\mathrm{Nb})$. Figure 10 shows behavior of thermophoresis parameter $(\mathrm{Nt})$ on concentration field $\phi(\eta)$. Here, concentration field is enhanced for larger thermophoresis parameter. Figure 11 elucidates the concentration for variation of Lewis number $(L e)$. Obviously larger $(L e)$ leads to a large concentration field. Figure 12 sketched the concentration field $\phi(\eta)$ against Prandtl number $(P r)$. Larger $(P r)$ shows concentration field. Figure 13 declares that the increasing values of squeezing parameter $(S q)$ lead to higher enhancement. Table 3 is developed to validate the present results with the previously published results in a limiting sense. From this Table, we analyzed that the present HAM solution have good agreement with the previous solution by Hashmi et al. [6] in a limiting sense. Table 4 consists of skin friction at the lower and upper disks. Here, the skin friction coefficient at the lower and upper disks are higher for increasing Deborah number and squeezing parameter. Table 5 is computed to examine the numerical data of local Nusselt number at the lower and upper disks for several embedding 
parameters. It is observed that local Nusselt number enhances at both lower and upper disks for larger Lewis number while the reverse is found for Prandtl number. Table 6 depicts numerical data of local Sherwood number at the lower and upper disks for various values of pertinent parameters. Here, we noticed that local Sherwood number increases at both lower and upper disks for increasing values of squeezing parameter.

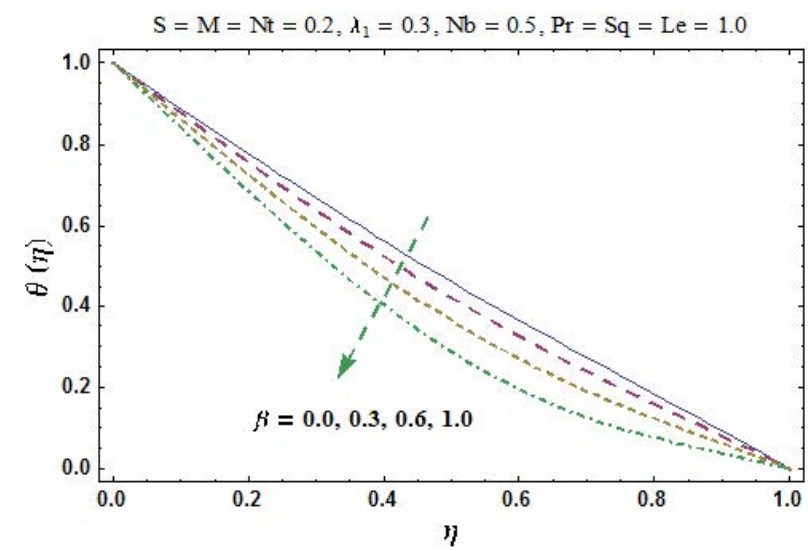

Figure 3. Plots of $\theta(\eta)$ for $\beta$.

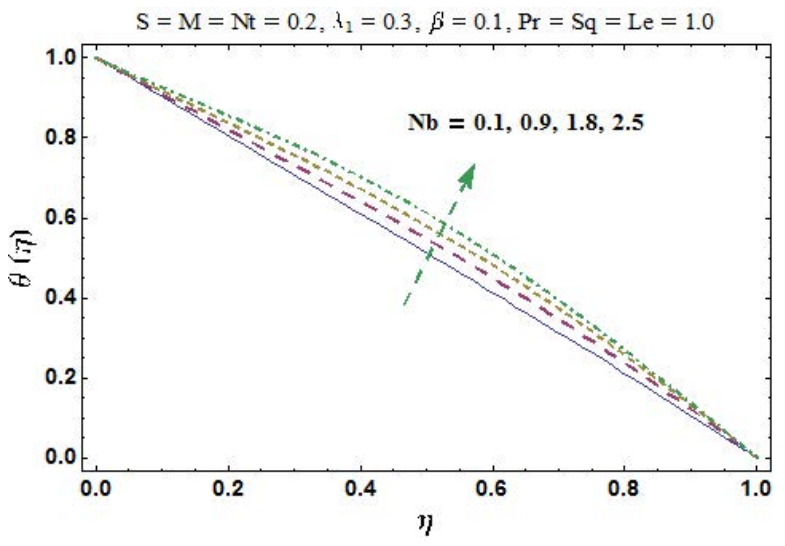

Figure 4. Plots of $\theta(\eta)$ for $N b$.

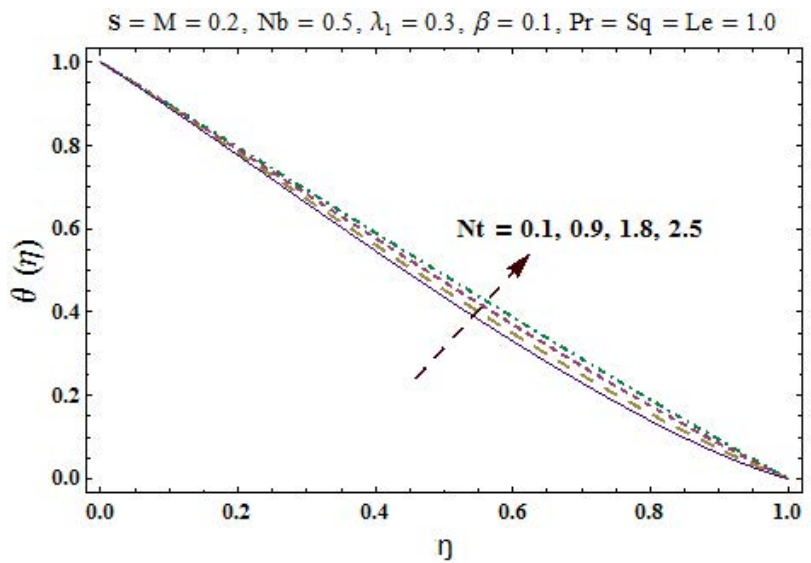

Figure 5. Plots of $\theta(\eta)$ for $N t$. 


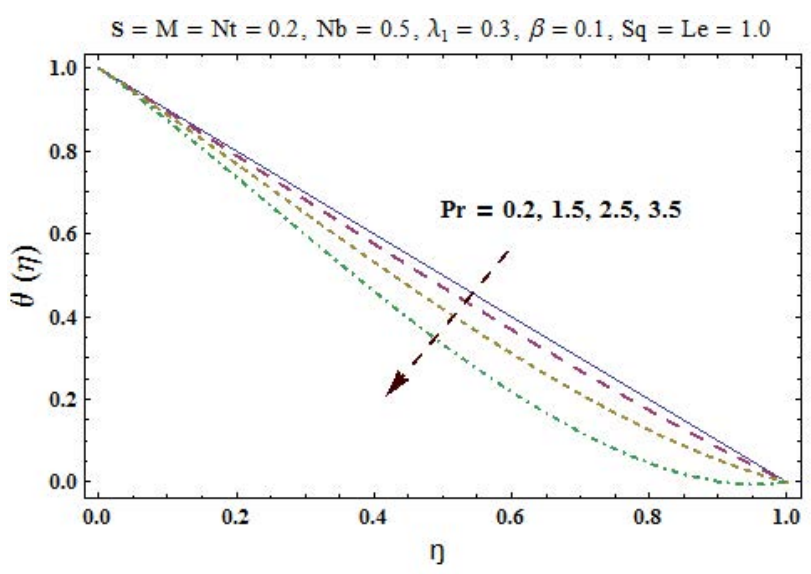

Figure 6. Plots of $\theta(\eta)$ for $P r$.

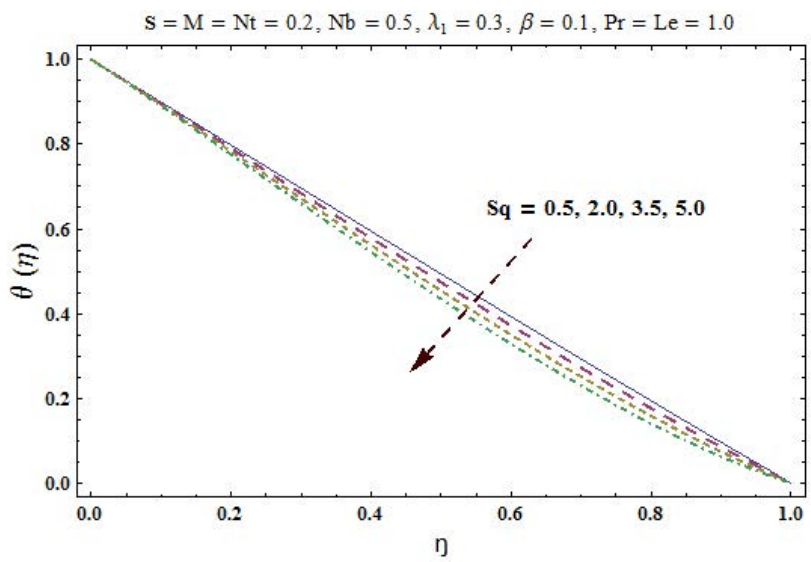

Figure 7. Plots of $\theta(\eta)$ for $S q$.

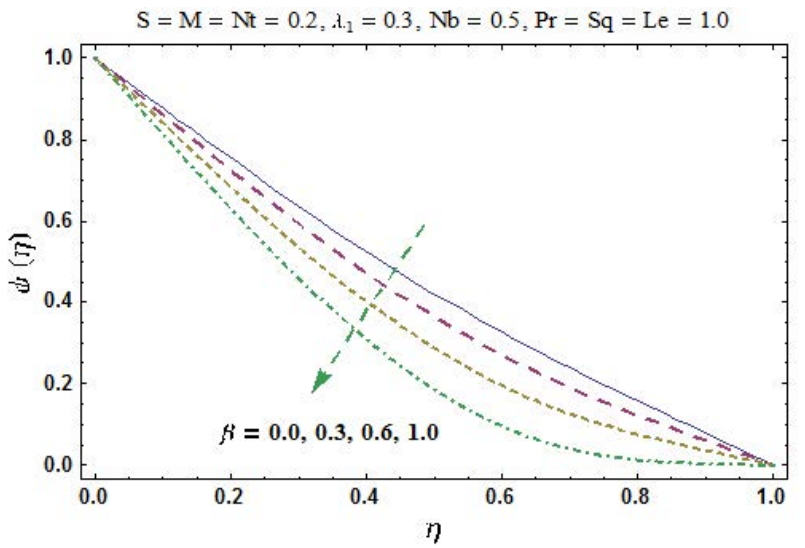

Figure 8. Plots of $\phi(\eta)$ for $\beta$. 


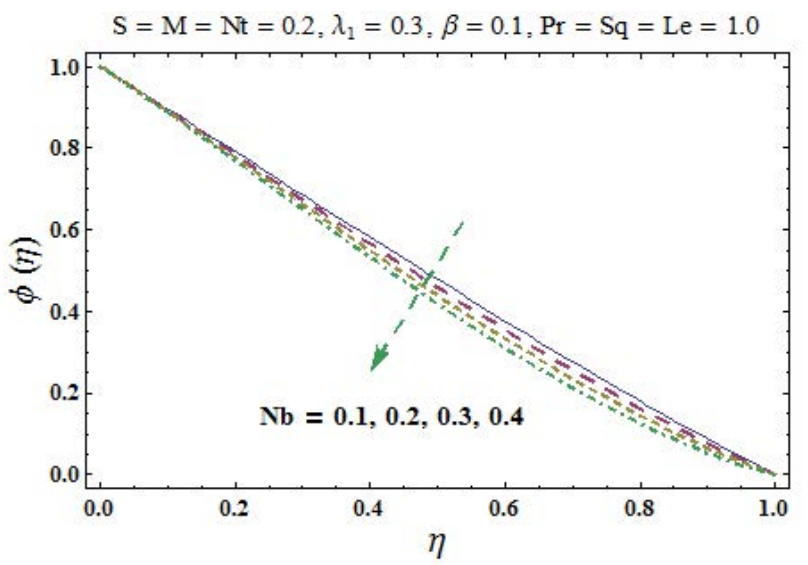

Figure 9. Plots of $\phi(\eta)$ for $N b$.

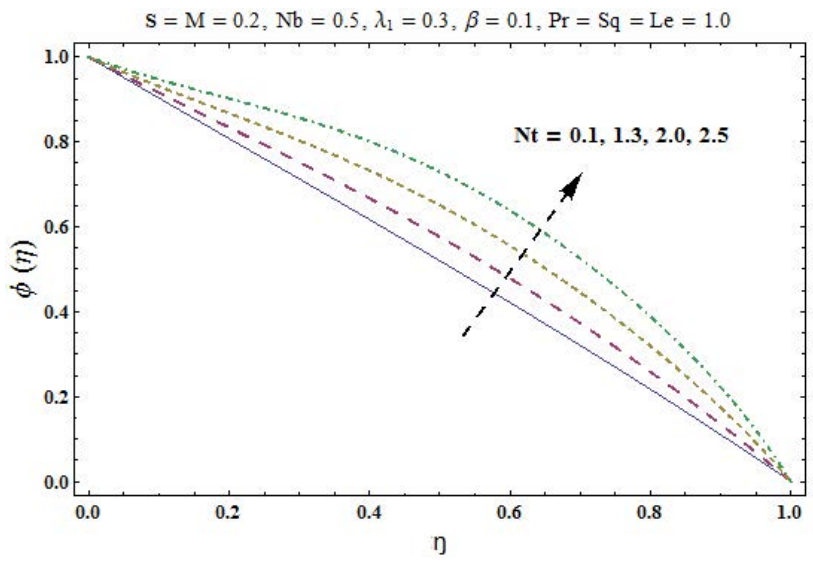

Figure 10. Plots of $\phi(\eta)$ for $N t$.

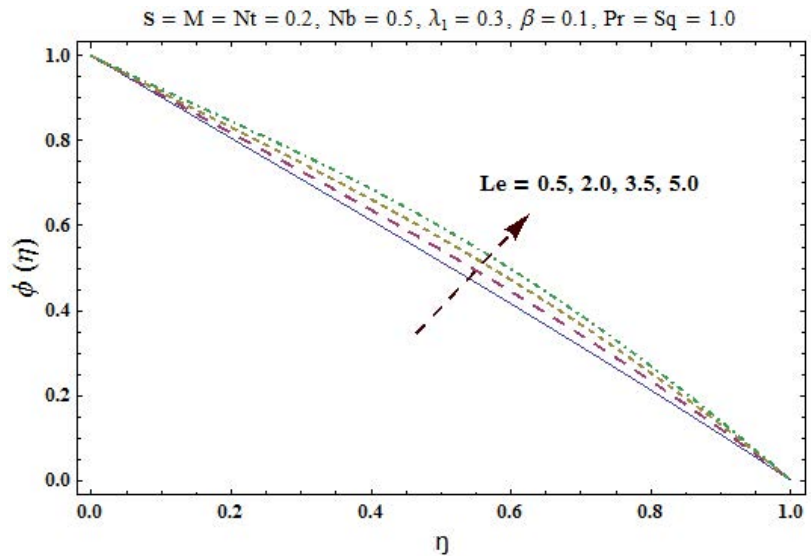

Figure 11. Plots of $\phi(\eta)$ for Le. 


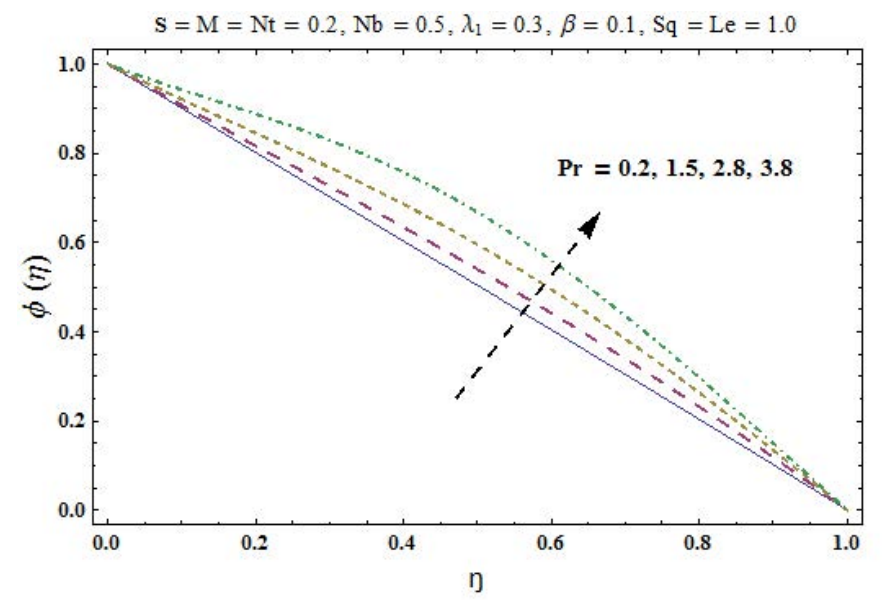

Figure 12. Plots of $\phi(\eta)$ for $P r$.

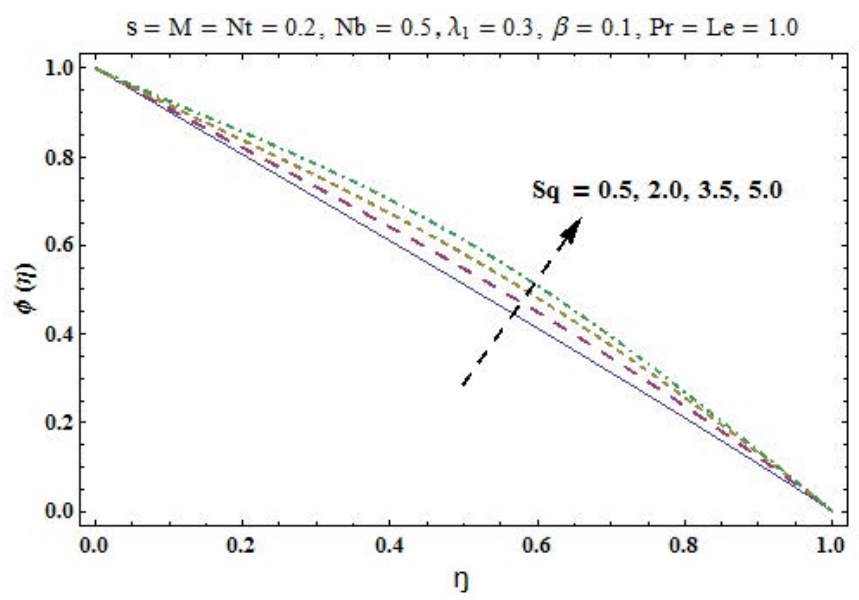

Figure 13. Plots of $\phi(\eta)$ for $S_{q}$.

Table 3. Comparative values of $f^{\prime \prime}(1)$ for different values of $M$ when $S q=1.0, S=2.0$ and $\beta=\lambda_{1}=0.0$.

\begin{tabular}{ccc}
\hline \multirow{2}{*}{$\boldsymbol{M}$} & \multicolumn{2}{c}{$\boldsymbol{f}^{\prime \prime}(\mathbf{1})$} \\
\cline { 2 - 3 } & HAM & Hashmi et al. [6] \\
\hline 0.0 & 7.533166 & 7.53316579 \\
2.0 & 8.263872 & 8.26387230 \\
3.0 & 9.097326 & 9.09732573 \\
5.0 & 11.34929 & 11.3492890 \\
\hline
\end{tabular}


Table 4. Skin friction coefficient at the lower and upper disks via $S, \beta, \lambda_{1}, M$ and $S q$.

\begin{tabular}{ccccccc}
\hline $\boldsymbol{S}$ & $\boldsymbol{\beta}$ & $\lambda_{\mathbf{1}}$ & $\boldsymbol{M}$ & $\boldsymbol{S q}$ & $\boldsymbol{C}_{\boldsymbol{f} \mathbf{1}}$ & $\boldsymbol{C}_{\boldsymbol{f} \mathbf{2}}$ \\
\hline 0.0 & 0.1 & 0.3 & 0.2 & 1.0 & 3.649479 & 3.590709 \\
0.2 & - & - & - & -- & 2.287085 & 2.097308 \\
0.4 & - & - & - & - & 0.795248 & 0.681540 \\
0.2 & 0.0 & 0.3 & 0.2 & 1.0 & 2.002700 & 1.851550 \\
- & 0.1 & - & - & - & 2.280654 & 2.092601 \\
- & 0.2 & - & - & - & 2.554007 & 2.344925 \\
0.2 & 0.1 & 0.0 & 0.2 & 1.0 & 2.232063 & 2.087393 \\
- & - & 0.1 & - & - & 2.248280 & 2.089086 \\
- & - & 0.2 & - & - & 2.264478 & 2.090822 \\
0.2 & 0.1 & 0.3 & 0.0 & 1.0 & 2.279427 & 2.091703 \\
- & - & - & 0.5 & - & 2.287085 & 2.097308 \\
- & - & - & 1.0 & - & 2.309896 & 2.114041 \\
0.2 & 0.1 & 0.3 & 0.2 & 0.5 & 2.175138 & 2.081827 \\
- & - & - & - & 1.0 & 2.280654 & 2.092601 \\
- & - & - & - & 1.5 & 2.385315 & 2.105140 \\
- & - & - & - & 2.0 & 2.489154 & 2.119203 \\
\hline
\end{tabular}

Table 5. Numerical data for local Nusselt number at the lower and upper disks for several values of $S$, $M, S q, N t, N b$, Le and $P r$.

\begin{tabular}{ccccccccc}
\hline$S$ & $\boldsymbol{M}$ & $S \boldsymbol{q}$ & $\boldsymbol{N} \boldsymbol{t}$ & $\mathrm{Nb}$ & $\mathbf{L e}$ & $\boldsymbol{P r}$ & $\boldsymbol{\theta}^{\prime}(\mathbf{0})$ & $\boldsymbol{\theta}^{\prime}(\mathbf{1})$ \\
\hline 0.0 & 0.2 & 1.0 & 0.2 & 0.5 & 1.0 & 1.0 & 0.77442 & 1.21503 \\
0.5 & - & - & - & - & - & - & 0.63707 & 1.28291 \\
1.0 & - & - & - & - & - & - & 0.52424 & 1.34412 \\
0.5 & 0.0 & 1.0 & 0.2 & 0.5 & 1.0 & 1.0 & 0.63707 & 1.28291 \\
- & 0.5 & - & - & - & - & - & 0.63707 & 1.28291 \\
- & 1.0 & - & - & - & - & - & 0.63707 & 1.28291 \\
0.5 & 0.2 & 0.0 & 0.2 & 0.5 & 1.0 & 1.0 & 0.69050 & 1.39050 \\
- & - & 1.0 & - & - & - & - & 0.63707 & 1.28291 \\
- & - & 2.0 & - & - & - & - & 0.58798 & 1.18403 \\
0.5 & 0.2 & 1.0 & 0.0 & 0.5 & 1.0 & 1.0 & 0.71036 & 1.17119 \\
- & - & - & 0.5 & - & - & - & 0.53789 & 1.46215 \\
- & - & - & 1.0 & - & - & - & 0.39954 & 1.79063 \\
0.5 & 0.2 & 1.0 & 0.2 & 0.5 & 1.0 & 1.0 & 0.71623 & 1.24304 \\
- & - & - & - & 1.0 & - & - & 0.54547 & 1.56082 \\
- & - & - & - & 1.5 & - & - & 0.40812 & 1.92538 \\
0.5 & 0.2 & 1.0 & 0.2 & 0.5 & 0.5 & 1.0 & 0.63610 & 1.28096 \\
- & - & - & - & - & 1.0 & - & 0.63707 & 1.28291 \\
- & - & - & - & - & 1.5 & - & 0.63816 & 1.28511 \\
0.5 & 0.2 & 1.0 & 0.2 & 0.5 & 1.0 & 0.5 & 0.80131 & 1.13712 \\
- & - & - & - & - & - & 1.0 & 0.63707 & 1.28291 \\
- & - & - & - & - & - & 1.5 & 0.50384 & 1.43981 \\
\hline
\end{tabular}


Table 6. Numerical data for local Sherwood number at the lower and upper disks for several values of $S, M, S q, N t, N b$, Le and $P r$.

\begin{tabular}{ccccccccc}
\hline$S$ & $\boldsymbol{M}$ & $S \boldsymbol{q}$ & $\boldsymbol{N} \boldsymbol{t}$ & $\boldsymbol{N b}$ & $\boldsymbol{L e}$ & $\boldsymbol{P r}$ & $\boldsymbol{\phi}^{\prime}(\mathbf{0})$ & $\boldsymbol{\phi}^{\prime}(\mathbf{1})$ \\
\hline 0.0 & 0.2 & 1.0 & 0.2 & 0.5 & 1.0 & 1.0 & 0.99838 & 1.10381 \\
0.5 & - & - & - & - & - & - & 1.23601 & 1.03275 \\
1.0 & - & - & - & - & - & - & 1.49070 & 0.96658 \\
0.5 & 0.0 & 1.0 & 0.2 & 0.5 & 1.0 & 1.0 & 1.23601 & 1.03275 \\
- & 0.5 & - & - & - & - & - & 1.23601 & 1.03275 \\
- & 1.0 & - & - & - & - & - & 1.23601 & 1.03275 \\
0.5 & 0.2 & 0.0 & 0.2 & 0.5 & 1.0 & 1.0 & 1.12380 & 0.84379 \\
- & - & 1.0 & - & - & - & - & 1.23601 & 1.03275 \\
- & - & 2.0 & - & - & - & - & 1.33266 & 1.19929 \\
0.5 & 0.2 & 1.0 & 0.0 & 0.5 & 1.0 & 1.0 & 1.07964 & 1.11899 \\
- & - & - & 0.5 & - & - & - & 1.58185 & 0.73579 \\
- & - & - & 1.0 & - & - & - & 2.39964 & 0.26741 \\
0.5 & 0.2 & 1.0 & 0.2 & 0.5 & 1.0 & 1.0 & 1.09150 & 1.07475 \\
- & - & - & - & 1.0 & - & - & 1.07083 & 1.10001 \\
- & - & - & - & 1.5 & - & - & 1.06200 & 1.12874 \\
0.5 & 0.2 & 1.0 & 0.2 & 0.5 & 0.5 & 1.0 & 1.19159 & 0.96141 \\
- & - & - & - & - & 1.0 & - & 1.23601 & 1.03275 \\
- & - & - & - & - & 1.5 & - & 1.27885 & 1.10206 \\
0.5 & 0.2 & 1.0 & 0.2 & 0.5 & 1.0 & 0.5 & 1.12319 & 1.00307 \\
- & - & - & - & - & - & 1.0 & 1.23601 & 1.03275 \\
- & - & - & - & - & - & 1.5 & 1.33897 & 1.08419 \\
\hline
\end{tabular}

\section{Conclusions}

Magnetohydrodynamic (MHD) squeezing flow of Jeffrey nanofluid between two parallel disks is examined. The key points of presented analysis are mentioned below:

- Larger values of Deborah number correspond to lower temperature and concentration profiles.

- Both temperature and concentration profiles are higher for larger values of thermophoresis parameter.

- Effects of Brownian motion parameter on temperature and concentration profiles are quite the opposite from each other.

- Larger values of Prandtl number show opposite trends for temperature and concentration profiles.

- Effects of squeezing parameter on temperature and concentration profiles are quite opposite to each other.

- The present analysis reduces to a Newtonian nanofluid flow situation when $\beta=\lambda_{1}=0$.

Acknowledgments: The authors are grateful for the useful suggestions of the reviewers.

Author Contributions: All authors contributed. Tasawar Hayat, Muhammad Ayub and Ahmed Alsaedi contributed in Sections 1, 2 and 5. Tehseen Abbas and Taseer Muhammad mainly have contribution in Sections 3 , 4 and 5.

Conflicts of Interest: The authors declare no conflict of interest.

\section{References}

1. Choi, S.U.S. Enhancing Thermal Conductivity of Fluids with Nanoparticles; FED 231/MD; ASME: New York, NY, USA, 1995; pp. 99-105.

2. Buongiorno, J. Convective transport in nanofluids. ASME J. Heat Transf. 2006, 128, 240-250.

3. Makinde, O.D.; Aziz, A. Boundary layer flow of a nanofluid past a stretching sheet with a convective boundary condition. Int. J. Therm. Sci. 2011, 50, 1326-1332.

4. Mustafa, M.; Hayat, T.; Pop, I.; Asghar, S.; Obaidat, S. Stagnation-point flow of a nanofluid towards a stretching sheet. Int. J. Heat Mass Transf. 2011, 54, 5588-5594. 
5. Turkyilmazoglu, M. Exact analytical solutions for heat and mass transfer of MHD slip flow in nanofluids. Chem. Eng. Sci. 2012, 84, 182-187.

6. Hashmi, M.M.; Hayat, T.; Alsaedi, A. On the analytic solutions for squeezing flow of nanofluid between parallel disks. Nonlinear Anal. Model. Control 2012, 17, 418-430.

7. Ibrahim, W.; Makinde, O.D. The effect of double stratification on boundary layer flow and heat transfer of nanofluid over a vertical plate. Comput. Fluids 2013, 86, 433-441.

8. Sheikholeslami, M.; Bandpy, M.G.; Ellahi, R.; Hassan, M.; Soleimani, S. Effects of MHD on Cu-water nanofluid flow and heat transfer by means of CVFEM. J. Magn. Magn. Mater. 2014, 349, 188-200.

9. Malvandi, A.; Safaei, M.R.; Kaffash, M.H.; Ganji, D.D. MHD mixed convection in a vertical annulus filled with $\mathrm{Al}_{2} \mathrm{O}_{3}$-Water nanofluid considering nanoparticle migration. J. Magn. Magn. Mater. 2015, 382, $296-306$.

10. Hayat, T.; Muhammad, T.; Alsaedi, A.; Alhuthali, M.S. Magnetohydrodynamic three-dimensional flow of viscoelastic nanofluid in the presence of nonlinear thermal radiation. J. Magn. Magn. Mater. 2015, 385, 222-229.

11. Chamkha, A.; Abbasbandy, S.; Rashad, A.M. Non-Darcy natural convection flow for non-Newtonian nanofluid over cone saturated in porous medium with uniform heat and volume fraction fluxes. Int. J. Numer. Methods Heat Fluid Flow 2015, 25, 422-437.

12. Gireesha, B.J.; Gorla, R.S.R.; Mahanthesh, B. Effect of suspended nanoparticles on three-dimensional MHD flow, heat and mass transfer of radiating Eyring-Powell fluid over a stretching sheet. J. Nanofluids 2015, 4, 474-484.

13. Lin, Y.; Zheng, L.; Zhang, X.; Ma, L.; Chen, G. MHD pseudo-plastic nanofluid unsteady flow and heat transfer in a finite thin film over stretching surface with internal heat generation. Int. J. Heat Mass Transf. 2015, 84, 903-911.

14. Sheikholeslami, M.; Ellahi, R. Three dimensional mesoscopic simulation of magnetic field effect on natural convection of nanofluid. Int. J. Heat Mass Transf. 2015, 89, 799-808.

15. Hsiao, K.L. Stagnation electrical MHD nanofluid mixed convection with slip boundary on a stretching sheet. Appl. Therm. Eng. 2016, 98, 850-861.

16. Hayat, T.; Muhammad, T.; Shehzad, S.A.; Alsaedi, A. On three-dimensional boundary layer flow of Sisko nanofluid with magnetic field effects. Adv. Powder Tech. 2016, 27, 504-512.

17. Malvandi, A.; Ganji, D.D.; Pop, I. Laminar filmwise condensation of nanofluids over a vertical plate considering nanoparticles migration. Appl. Therm. Eng. 2016, 100, 979-986.

18. Hayat, T.; Imtiaz, M.; Alsaedi, A. Unsteady flow of nanofluid with double stratification and magnetohydrodynamics. Int. J. Heat Mass Transf. 2016, 92, 100-109.

19. Hayat, T.; Waqas, M.; Shehzad, S.A.; Alsaedi, A. On model of Burgers fluid subject to magneto nanoparticles and convective conditions. J. Mol. Liq. 2016, 222, 181-187.

20. Hayat, T.; Aziz, A. ; Muhammad, T.; Alsaedi, A. On magnetohydrodynamic three-dimensional flow of nanofluid over a convectively heated nonlinear stretching surface. Int. J. Heat Mass Transf. 2016, 100, 566-572.

21. Hussain, A.; Mohyud-Din, S.T.; Cheema, T.A. Analytical and numerical approaches to squeezing flow and heat transfer between two parallel disks with velocity slip and temperature jump. Chin. Phys. Lett. 2012, 29, 114705.

22. Chatraei, S.H.; Macosko, C.W.; Winter, H.H. Lubricated squeezing flow: A new biaxial extensional rheometer. J. Rheol. 1981, 25, 433-443.

23. Stefan, M.J. Versuch Uber die scheinbare adhesion, Sitzungsberichte der Akademie der Wissenschaften in Wien. Math. Naturwissen 1874, 69, 713-721.

24. Domairry, G.; Aziz, A. Approximate analysis of MHD squeeze flow between two parallel disks with suction or injection by homotopy perturbation method. Math. Prob. Eng. 2009, 2009, 603916.

25. Siddiqui, A.M.; Irum, S.; Ansari, A.R. Unsteady squeezing flow of a viscous MHD fluid between parallel plates, a solution using the homotopy perturbation method. Math. Model. Anal. 2008, 13, 565-576.

26. Rashidi, M.M.; Siddiqui, A.M.; Asadi, M. Application of homotopy analysis method to the unsteady squeezing flow of a second-grade fluid between circular plates. Math. Probl. Eng. 2010, 2010, 706840.

27. Qayyum, A.; Awais, M.; Alsaedi, A.; Hayat, T. Unsteady squeezing flow of Jeffery fluid between two parallel disks. Chin. Phys. Lett. 2012, 29, 034701. 
28. Sheikholeslami, M.; Ganji, D.D. Heat transfer of $\mathrm{Cu}$-water nanofluid flow between parallel plates. Powder Technol. 2013, 235, 873-879.

29. Hayat, T.; Muhammad, T.; Qayyum, A.; Alsaedi, A.; Mustafa, M. On squeezing flow of nanofluid in the presence of magnetic field effects. J. Mol. Liq. 2016, 213, 179-185.

30. Hayat, T.; Mustafa, M.; Shehzad, S.A.; Obaidat, S. Melting heat transfer in the stagnation-point flow of an upper-convected Maxwell (UCM) fluid past a stretching sheet. Int. J. Numer. Methods Fluids 2012, 68, 233-243.

31. Liao, S.J. On the homotopy analysis method for nonlinear problems. Appl. Math. Comput. 2004, 147, 499-513.

32. Dehghan, M.; Manafian, J.; Saadatmandi, A. Solving nonlinear fractional partial differential equations using the homotopy analysis method. Numer. Methods Part. Differ. Equ. 2010, 26, 448-479.

33. Malvandi, A.; Hedayati, F.; Domairry, G. Stagnation point flow of a nanofluid toward an exponentially stretching sheet with nonuniform heat generation/absorption. J. Thermodyn. 2013, 2013, 764827.

34. Abbasbandy, S.; Hashemi, M.S.; Hashim, I. On convergence of homotopy analysis method and its application to fractional integro-differential equations. Quaest. Math. 2013, 36, 93-105.

35. Arqub, O.A.; El-Ajou, A. Solution of the fractional epidemic model by homotopy analysis method. J. King Saud Univ. Sci. 2013, 25, 73-81.

36. Ellahi, R.; Hassan, M.; Zeeshan, A. Shape effects of nanosize particles in $\mathrm{Cu}-\mathrm{H}_{2} \mathrm{O}$ nanofluid on entropy generation. Int. J. Heat Mass Transf. 2015, 81, 449-456.

37. Hayat, T.; Imtiaz, M.; Alsaedi, A. Impact of magnetohydrodynamics in bidirectional flow of nanofluid subject to second order slip velocity and homogeneous-Heterogeneous reactions. J. Magn. Magn. Mater. 2015, 395, 294-302.

38. Sui, J.; Zheng, L.; Zhang, X.; Chen, G. Mixed convection heat transfer in power law fluids over a moving conveyor along an inclined plate. Int. J. Heat Mass Transf. 2015, 85, 1023-1033.

39. Hayat, T.; Hussain, Z.; Muhammad, T.; Alsaedi, A. Effects of homogeneous and heterogeneous reactions in flow of nanofluids over a nonlinear stretching surface with variable surface thickness. J. Mol. Liq. 2016, 221, 1121-1127.

40. Hayat, T.; Abbas, T.; Ayub, M.; Farooq, M.; Alsaedi, A. Flow of nanofluid due to convectively heated Riga plate with variable thickness. J. Mol. Liq. 2016, 222, 854-862.

41. Hsiao, K.L. MHD mixed convection for viscoelastic fluid past a porous wedge. Int. J. Non-Linear Mech. 2011, $46,1-8$.

42. Hsiao, K.L. Combined electrical MHD heat transfer thermal extrusion system using Maxwell fluid with radiative and viscous dissipation effects. Appl. Therm. Eng. 2016, 8, 208.

43. Hsiao, K.L. Numerical solution for Ohmic Soret-Dufour heat and mass mixed convection of viscoelastic fluid over a stretching sheet with multimedia physical features. J. Aerosp. Eng. 2016, doi:10.1061/(ASCE)AS.1943-5525.0000681.

(C) 2016 by the authors; licensee MDPI, Basel, Switzerland. This article is an open access article distributed under the terms and conditions of the Creative Commons Attribution (CC-BY) license (http://creativecommons.org/licenses/by/4.0/). 